Pulsar Astronomy -- 2000 and Beyond

ASP Conference Series, Vol. 202, 2000

M. Kramer, N. Wex, and R. Wielebinski, eds.

\title{
How well are Neutron Stars understood?
}

\author{
Wolfgang Kundt
}

Inst. f. Astroph. der Universität, Auf dem Hügel 71, D-53121 Bonn

\begin{abstract}
Alternative ways are discussed in which pulsars may (1) drive their winds, (2) avoid net electric currents, (3) emit coherent radio pulses, (4) acquire high spin rates, and (5) deal with moderate surface magnetic fields: $10^{11}<B / G \lesssim 10^{14}$.
\end{abstract}

(1) How strong are pulsar winds? Does the Goldreich-Julian charge density escape at the speed of light from the two polar caps, in the form of electrons and positrons, or are pulsar winds yet some $\xi \approx 10^{4}$-times stronger? There is the theoretical argument that only electric forces are strong enough to overcome a neutron star's gravity, that they would act preferentially on the (light) leptons, and that they should act in an AC mode in order to avoid excessive spa ce charges, both on the neutron star, and on its corotating magnetosphere. The 'primary' $\mathrm{AC}$ current will have strong inverse-Compton and curvature losses, and the resulting (hard) photons create the legendary secondary pairs, via Erber's scattering on the transverse magnetic field. In this way, a $\xi$-fold overproduction of pairs can give rise to a strong charge-neutral, current-free wind which reaches the speed-of-light cylinder unbraked (except for radiative losses), where the strong outgoing magnetic-dipole wave takes care of powerful post-acceleration (Kundt \& Schaaf, 1993). This scheme predicts winds of order $\xi$-times Goldreich-Julian, whose high densities help provide the large coherence (in the spikes) of pulsar radio emission. Independently, such high wind densities are demanded by the large radii of (mapped) standoff-bowshocks around at least nine pulsars, of size $\lesssim 10^{18} \mathrm{~cm}$ (Kundt, 1998). Note that the small general-relativistic corrections to the driving polar-cap voltages, highlighted by Muslimov \& Tsygan (1992), do not change this scenario qualitatively.

A problem with high wind densities (of pulsars) has been pointed out by Kunzl et al (1998), also by Harald Lesch at this colloquium: Plasma densities in the (expected) emission region are so high that the local plasma frequency exceeds the frequencies to be emitted. This does no harm to our scenario in which the escaping plasma layers (chapátis) owe their density modulation to their genesis at a polar cap plus radiative braking $\left(\sim \gamma^{4}\right)$ of the fastest. And their high plasma frequencies are those of the antennae, not of the medium of propagation. Besides, there is the magneto-acoustic branch.

(2) Do pulsar winds carry net electric currents? If they did, such currents would have to close via return currents, at lower latitudes (Arons, 1998), and further as Pedersen currents inside the neutron star, across its magnetic fields. Implied Hall currents would be $10^{4 \pm 1}$-times stronger (Kundt \& Schaaf, 1993), and the stellar surface layers would be heated to hard X-ray temperatures, in conflict with the observations (if currents were of Goldreich-Julian order). This 
estimate follows from the fact that the voltage across a polar cap causes the charges to $\vec{E} \times \vec{B}$-drift along latitude circles; they can only fall 'inward', parallel to the electric field, and thereby close the current loop whenever they transiently deposit their kinetic energy via a collision, or via radiation, so that the Lorentz force is switched off. During such free-falls, they regain their kinetic energy; the polar-cap's electrostatic energy is thus converted to heat.

(3) How do pulsars emit their strong radio pulses? Don Melrose has been efficient over the decades to invalidate various proposals, cf. Melrose \& Gedalin (1998), but at this colloquium, he did not keep his (oral) promise to shoot down mine (Kundt, 1998): On their way out through the corotating magnetosphere, the escaping electrons and positrons are forced to (almost) follow the guiding magnetic field lines. But whilst centrifugal forces increase with distance, magnetic guiding forces decrease, and at a critical distance - the emission height - (damped) gyrations are coherently excited. They cause the charges to emit small-pitch-angle synchro-curvature radiation, as a minimum radiative loss.

(4) Are all pulsars born in core-collapse supernovae, or have the fast ones - the msec hump of the bimodal period histogram - been 'recycled' via mass transfer from a binary companion? In (Kundt, 1998), I have given a number of reasons for why I do not trust the feasibility of recycling: Spinup via accretion is counteracted by spindown via disk friction and wind thrust, leading to oscillatory excursions around stable steady-state spin rates in all the observed X-ray binaries. There is a complete absence of (long-lived!) accelerating progenitor systems in the Galaxy; msec pulsars are the likely result of supernovae with less-than-average magnetic dipole coupling of the core to the overlying stellar envelope. They can be much younger than commonly assumed.

(5) How strong are the surface magnetic fields of pulsars? After what has been said, blowing a strong wind requires magnetic fields in excess of $10^{11} \mathrm{G}$, in order to allow for the production of secondary pairs (via Erber's process). Apparently, the surface field can be orders of magnitude higher than the transverse dipole component inferred from the spindown rate. On the other hand, I mistrust fields much in excess of (the transverse-dipole record) $10^{13.74} \mathrm{G}$, as postulated for the so-called magnetars (Thompson \& Duncan, 1996): such estimates arrive at implausibly high luminosities via unrealistically large distance estimates (for the SGRs): Kundt \& Chang (1994).

\section{References}

Arons, J. 1998, in Neutron Stars and Pulsars, eds. Shibazaki et al, 339-349

Kunzl, T., Lesch, H., Jessner, A., \& von Hoensbroech, A. 1998, ApJ 505, L139

Kundt, W. 1998, Fundam. Cosm. Phys. 20, 1-119

Kundt, W., \& Chang, H.-K. 1994, in AIP Conf. Proc. 307, Gamma-Ray Bursts, eds. G.J. Fishman, J.J. Brainerd, \& J.K. Hurley (New York: AIP), 596

Kundt, W., \& Schaaf, R. 1993, Ap\&SS 200, 251

Melrose, D.B., \& Gedalin, M.E. 1999, ApJ 521, 351

Muslimov, A.G., \& Tsygan, A.I. 1992, MNRAS 255, 61

Thompson, C., \& Duncan, R. 1996, ApJ 473, 322 